\title{
Prognostic Significance of Long Non Coding RNAANRIL and SNHG14 in Acute Myeloid Leukemia
}

\author{
Marwa A Gamaleldin ${ }^{1 *}$, Omar Ghallab ${ }^{2}$, Reham A Abo Elwafa ${ }^{1}$
}

\begin{abstract}
Background: Long non-coding RNAs (lnRNAs) play a pivotal role in various malignancies including AML. Therefore, we decided to study both lnRNA ANRIL and lnRNA SNHG14 gene expressions in patients with AML to better understand their role in AML risk development, clinical presentation, and prognosis. Methods: The current prospective study included two hundred participants, 100 AML patients and 100 control subjects. Bone marrow analysis was made to all patients, in addition to gene expression molecular testing of both lnRNA ANRIL and lnRNA SNHG14. Results: Both lnRNA SNHG14 and lnRNA ANRIL showed high expression levels in AML bone marrow samples compared to non-AML subjects and were remarkably associated with lower Complete Remission (OR: 3.449, 95\% CI: $1.324-8.985, \mathrm{p}=0.011$ for ANRIL and OR: $3.955,95 \%$ CI: $1.510-10.356, p=0.005$ for SNHG14), Relapse Free Survival $(\mathrm{HR}=3.504,95 \% \mathrm{CI}$ : $1.662-7.387, \mathrm{p}=0.001$ for ANRIL and HR=4.094, 95\%CI: $1.849-9.067, \mathrm{p}=0.001$ for SNHG14) and Overall Survival (HR=3.353, 95\%CI: 1.434-7.839, $\mathrm{p}=0.005$ for ANRIL and $\mathrm{HR}=3.094,95 \% \mathrm{CI}$ : 1.277-7.494, $\mathrm{p}=0.012$ for SNHG14), favouring poor prognostic significance in AML. Conclusion: This suggests that both lnRNA ANRIL and lnRNA SNHG14 could be used in the future as prognostic biomarkers to help in treatment decisions and follow up of AML patients.
\end{abstract}

Keywords: lnRNA- ANRIL- SNHG14- AML- gene expression

Asian Pac J Cancer Prev, 22 (12), 3763-3771

\section{Introduction}

AML, a common type of leukemia, is caused by uncontrolled proliferation of immature clonal hematopoietic myeloid progenitors in the bone marrow and peripheral blood and hemopoietic organs as liver and spleen (Döhner et al., 2015; Prada-Arismendy et al., 2017; Shallis et al., 2019). Despite recent technologies and treatment modalities, still most patients with AML suffer from side effects of chemotherapy, cancer recurrence and high rates of mortalities, leading to unsatisfactory overall prognosis (Döhner et al., 2015; Kadia et al., 2016; Gamaleldin and Imbaby, 2021). AML is a highly heterogenous malignancy, rendering prediction of prognosis extremely difficult. Despite that cytogenetic analysis has provided the best way for risk stratification of AML for a long time, yet it was not satisfactory in about $50 \%$ of the AML cases who experienced normal karyotyping with different outcomes (Abo Elwafa et al., 2019). Hence, to help develop better prognosis and treatment options for AML patients, it is required to identify new biomarkers to improve diagnosis, prognosis, and treatment results.

As the Human Genome Project showed that about $98 \%$ of the 3 billion base pairs that make up the human genome are non-protein coding sequences (Wang and Wen, 2020), non-coding RNAs started to gain more attention. Non-coding RNAs are divided into small and long non-coding RNAs according to their transcript length. lnRNAs show a length of more than 200bp with inability to encode proteins (Ferrè et al., 2016; Jathar et al., 2017; Wang and Wen, 2020).

Long non-coding RNAs (lnRNAs) play a pivotal role in gene transcription, post-transcription and translational levels, thus affecting cell proliferation, differentiation and survival (Ferrè et al., 2016; Jathar et al., 2017; Wang and Wen, 2020). Moreover, InRNAs regulate many biological and pathological functions in various cancers, including leukaemia (Bhan et al., 2017; Cruz-Miranda et al., 2019; Gugnoni and Ciarrocchi, 2019).

InRNAs were found by previous studies to modulate pathological functions in various malignancies including AML. For instance, lnRNA antisense non-coding RNA in the INK4 locus (ANRIL) which is located on chromososme 9p21, has been proved to be associated with cancers like melanoma (Pasmant et al., 2007; Congrains et al., 2013), laryngeal squamous cell carcinoma (Hao et al., 2019) and hepatocellular carcinoma (Ma et al., 2019). It is assumed that InRNA ANRIL has a specific role in AML including stimulating cell proliferation, migration, 
and invasion, in addition to inhibiting cell apoptosis by affecting miRNA-34a, histone deacetylase I and ASPP2 expressions (Wang et al., 2020). Moreover, InRNAANRIL facilitates cancer cell survival through activating glucose metabolic pathway including adiponectin receptor 1 (AdipoR1)/AMP-activated protein kinase (AMPK)/sirtuin (SIRT1) in AML (Sun et al., 2018).

Another lnRNA, called Small nucleolar RNA host gene (SNHG) 14 has been found by previous studies to play a role as an essential regulator of cellular processes in multiple types of human malignancies. It has been proved to stimulate oncogenesis by modulating the proliferation, migration and invasion of malignant cells, including non-small cell lung carcinoma (Wu et al., 2019), cervical (Zhang et al., 2019a) and gastric carcinoma (Liu et al., 2018a). For example, SNHG14 promotes cell proliferation via sponging miR-340 in NSCLC cells (Zhang et al., 2019b). Other studies reported that SNHG14 could contribute to the malignant process in breast cancer by modulating miR-193b-3p. However, its role in AML development and prognosis is still unclear.

Currently, there is not enough information on InRNAs in AML. Therefore, we decided to further study two lnRNAs in AML to explore new prognostic and therapeutic targets for this disease.

From this context, we decided to study both $\operatorname{lnRNA}$ ANRIL and lnRNA SNHG14 in patients with AML in order to better understand the role of these lnRNAs in AML risk development, clinical presentation, and prognosis.

\section{Materials and Methods}

Upon approval of the Medical Ethics Committee of Alexandria Faculty of Medicine, we recruited 100 newly diagnosed AML patients who visited the hematology unit in Alexandria Main University Hospitals (Alexandria, Egypt) from 2018 to 2020. 100 control subjects of matching age and sex to the patients' group, suffering from primary immune thrombocytopenia, hypersplenism or iron deficiency anemia were recruited as the control group. The sample size was calculated using the $\mathrm{G}$ power version 3.1 statistical software program with 0.05 level of significance and $80 \%$ power of the study. All study subjects signed an informed consent showing the nature and type of the study.

All AML patients were newly diagnosed primary AML. Complete blood count (CBC), bone marrow aspiration and/or biopsy, morphology, immunophenotyping, cytogenetics and molecular testing were done. The diagnosis was based on the FAB classification and WHO criteria. We excluded patients with acute promyelocytic leukemia (M3), secondary or relapsed AML and those patients with cytogenetic abnormalities. The control subjects were subjected to CBC, blood film morphology and $\operatorname{lnRNAANRIL}$ and $\operatorname{lnRNA}$ SNHG14 gene expression analysis using real-time PCR.

\section{Treatment Protocol}

Patients with AML were managed in concordance with the treatment strategy of the hematology Unit in
Alexandria University Hospitals. AML patients $\leq 60$ years of age took daunorubicin $45-60 \mathrm{mg} / \mathrm{m}^{2}$ for 3 days, followed by Ara-C $100 \mathrm{mg} / \mathrm{m}^{2}$ for 7 days. Assessment of the response was performed 21 to 28 days after induction therapy from bone marrow examination. If Complete Remission (CR) was attained, patients received 4 cycles of consolidation as high dose cytarabine. If no complete remission (NCR) was reached, the treatment protocol was repeated. If no response, the protocol was changed to high dose chemotherapy. For patients above 60 years of age, the treatment was evaluated by the treating physician according to the patient's circumstances (Kuendgen and Germing, 2009). For follow up after remission, all patients were examined every 1-3 months for the first 2 years and every 3-6 months subsequently by bone marrow examination.

Complete Remission (CR) was determined when neutrophil count was normalised (at least $\geq 1.5 \times 10^{9} / \mathrm{L}$ ) and platelet count $\left(>100 \times 10^{9} / \mathrm{L}\right)$, as well as bone marrow analysis showed at least $20 \%$ cellularity, $<5 \%$ blasts and absent Auer rods, as well as absence of extramedullary infiltration (Döhner et al., 2010). The overall survival (OS) was defined as the time elapsed from the first day of initial therapy to the date of death. Relapse free survival (RFS) was calculated from the date of CR to the date of relapse or death.

\section{Detection of $\ln R N A$ ANRIL}

Mononuclear cells (MCs) were extracted from the peripheral blood or bone marrow by densitygradient centrifugation. RNA was extracted from the mononuclear cells via QIAamp RNA Blood Mini kits (Qiagen, USA) according to the manufacturer's steps. The quality and quantity of the extracted RNA were measured by Nanodrop 2000 spectrophotometer (USA). The reverse transcription of the RNA was done by the High-capacity cDNA Reverse Transcription kit (Applied Biosystems, USA) and the PCR amplification was done using SimpliAmp Thermal Cycler (Applied Biosystems, USA). The primers used were: lnRNAANRIL 5'-TGCTCTATCCGCCAATCAGG-3' as the forward primer and 5'-GGCCTCAGTGGCACATACC-3' as the reverse primer. The PCR amplification program was: $95 \mathrm{C}$ for 5 seconds followed by 40 cycles of $95 \mathrm{C}$ for 5 seconds and $60 \mathrm{C}$ for 30 seconds. GADPH gene was used as the endogenous control with its expression being stable in all samples independent of the analysed variables. The relative expression of InRNAANRIL was analysed using the $2^{\Delta \Delta \mathrm{Ct}}$ formula (Livak and Schmittgen, 2001).

\section{Detection of $\ln R N A$ SNHG14}

Mononuclear cells (MCs) were extracted from the peripheral blood or bone marrow by density-gradient centrifugation. RNA is extracted from the mononuclear cells using QIAamp RNA Blood Mini kits (Qiagen, USA) according to the manufacturer's steps. The quality and quantity of the extracted RNA were measured by Nanodrop 2000 spectrophotometer (USA). The reverse transcription of the RNA was done by the High-capacity cDNA Reverse Transcription kit (Applied Biosystems, USA) and the PCR amplification was done using 
SimpliAmp Thermal Cycler (Applied Biosystems, USA). The primers used were: IncRNA SNHG14: 5'-GGGTGTTTACGTAGACCAGAACC-3' as the forward primer and 5'-CTTCCAAAAGCCTTCTGCCTTAG-3' as the reverse primer. The PCR amplification program was: $95 \mathrm{C}$ for minutes followed by 40 cycles of $95 \mathrm{C}$ for 10 seconds, $60 \mathrm{C}$ for 20 seconds and $72 \mathrm{C}$ for 34 seconds. GADPH gene was used as the endogenous control with its expression being stable in all samples independent of the analysed variables. The relative expression of $\operatorname{lnRNA}$ ANRIL was analysed using the $2 \Delta \Delta \mathrm{Ct}$ formula (Livak and Schmittgen, 2001).

\section{Statistical Analysis}

SPSS sortware version 22.0 was used for statistical analysis of data. Variations of mRNA expression of lnRNA ANRIL and InRNA SNHG14 between AML patients and controls was determined by the Wilcoxon test. For correlating lnRNAANRIL and lnRNA SNHG14 with clinical manifestations, patients were classified into high expression and low expression groups according to the median value of the respective lnRNA relative expression in AML patients. Comparing clinical features between high and low InRNA expression groups was performed by chi square $\left(X^{2}\right)$, Fisher's exact and Wilcoxon rank tests. A ROC curve was used to assess the value of lnRNA ANRIL and lnRNA SNHG14 to differentiate between AML patients and controls. Kaplan-Meier curves were used to assess the RFS and OS. To assess the difference of RFS and OS between high and low expression groups of both lnRNA genes, the log-rank test was used. Factors influencing RFS and OS were tested by univariate and multivariate regression analysis. Values of $p<0.05$ were considered statistically significant.

\section{Results}

Characteristics of the study subjects

In the control group, the mean age was $52.6 \pm 12.5$ years, with $22 \%$ females and $78 \%$ males. In AML patients'

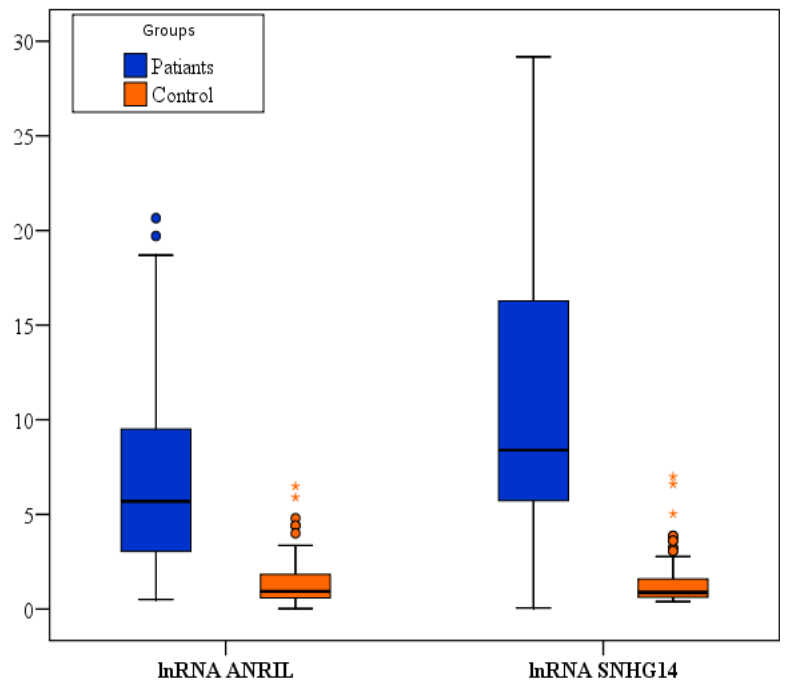

Figure 1. Relative Expression Levels of, InRNA ANRIL and B, InRNA SNHG14 in AML patients and non-malignant controls group, the mean age was $50.6 \pm 11.4$ with $33 \%$ females and $67 \%$ males. In terms of FAB classification, $17(17 \%)$, 16 (16\%), 19 (19\%), 0 (0\%), 20 (20\%), 25(25\%), 1(1\%) and $2(2 \%)$ patients were classified as M0, M1, M2, M3, M4, M5, M6 and M7. Regarding molecular genetics testing, $37(37 \%)$ and $40(40 \%)$ AML patients showed internal tandem duplications in FMS-like tyrosine kinase 3 (FLT3-ITD) and Nucleophosmin 1 (NPM1), respectively. Moreover, the median WBC count was 30.5 (0.3-302) x10 ${ }^{9}$ cells/1 in AML patients' group, compared to 6.6 (4-10.2) $\mathrm{x} 10^{9}$ cells $/ 1$ in the controls (Table 1 ).

Target gene expression levels and clinical criteria at diagnosis

There were significantly higher levels of ANRIL gene expression in AML patients compared with non-AML controls with medians of 5.7 versus 0.9 $(p<0.001)$. Similarly, SNHG14 gene expression was significantly increased in patients compared with controls with medians of 8.4 in AML versus 0.9 in controls $(\mathrm{p}<0.001)$ (Table 1, Figure 1).

No significant association was found between the levels of expression of either ANRIL or SNHG14 genes and other data including age, gender, FAB classification, and WBC counts (Table 2).

ROC curve analysis showed that $\ln$ RNAANRIL could differentiate AML patients from non-malignant controls (AUC: $0.924,95 \%$ CI: 0.887-0.960, $\mathrm{p}<0.001$ ), with a sensitivity of $83 \%$ and a specificity of $90 \%$ at the best cut-off point (Figure 2, Table 3). Similarly, ROC curve analysis showed that lnRNA SNHG14 was capable of differentiating AML patients from non-malignant controls (AUC: $0.919,95 \%$ CI: 0.872-0.965, $\mathrm{p}<0.001$ ), with a sensitivity of $87 \%$ and a specificity of $97 \%$ at the best cut-off point (Figure 2, Table 3).

Target gene expression levels and molecular genetics

InRNA ANRIL was significantly associated with positive NPM1 mutation ( $\mathrm{p}=0.004)$, while no association with FLT3/ITD mutation $(p=0.147)$ in patients with AML

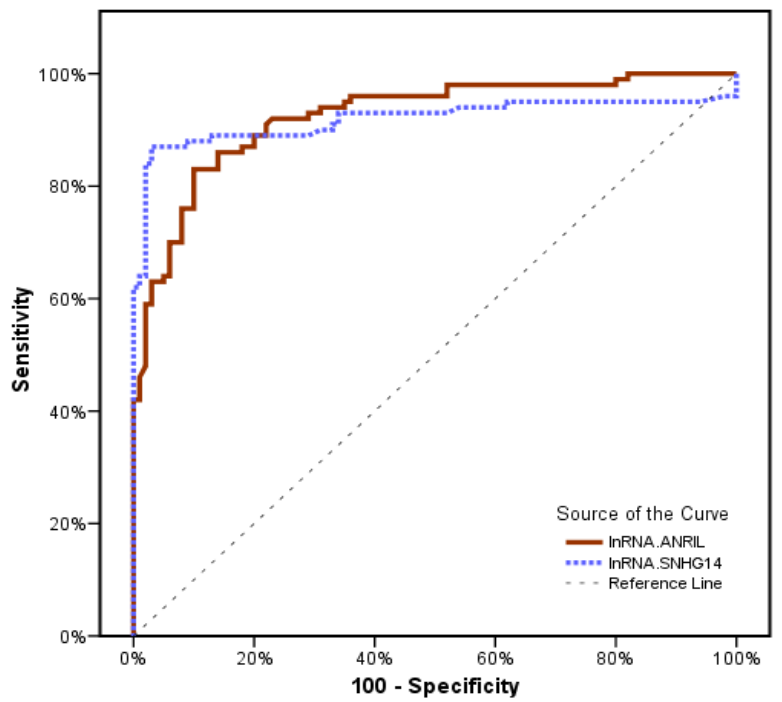

Figure 2. ROC Curve for lnRNA ANRIL and lnRNA SNHG14 to Predict AML Patients vs Control 
Table 1. Clinicopathological Findings of the Study Participants

\begin{tabular}{|c|c|c|c|}
\hline & $\begin{array}{l}\text { AML patients } \\
(\mathrm{n}=100)\end{array}$ & $\begin{array}{l}\text { Controls } \\
(\mathrm{n}=100)\end{array}$ & $\mathrm{p}$ \\
\hline \multicolumn{4}{|l|}{ Age } \\
\hline$<60$ & $78(78 \%)$ & $73(73 \%)$ & 0.411 \\
\hline$\geq 60$ & $22(22 \%)$ & $27(27 \%)$ & \\
\hline Mean $\pm \mathrm{SD}$ & $50.6 \pm 11.4$ & $52.6 \pm 12.5$ & 0.127 \\
\hline Median (Min. - Max.) & $53(22-79)$ & $54.5(24-76)$ & \\
\hline \multicolumn{4}{|l|}{ Gender } \\
\hline Male & $67(67 \%)$ & $78(78 \%)$ & 0.082 \\
\hline Female & $33(33 \%)$ & $22(22 \%)$ & \\
\hline \multicolumn{4}{|l|}{ WBC count $* 109 / 1$} \\
\hline Mean $\pm \mathrm{SD}$ & $45.3 \pm 53.6$ & $6.5 \pm 1.6$ & $<0.001 *$ \\
\hline Median (Min. - Max.) & $30.5(0.3-302)$ & $6.6(4-10.2)$ & \\
\hline \multicolumn{4}{|l|}{ FAB classification } \\
\hline M0 & $17(17 \%)$ & - & - \\
\hline M1 & $16(16 \%)$ & - & \\
\hline M2 & $19(19 \%)$ & - & \\
\hline M3 & $0(0 \%)$ & - & \\
\hline M4 & $20(20 \%)$ & - & \\
\hline M5 & $25(25 \%)$ & - & \\
\hline M6 & $1(1 \%)$ & - & \\
\hline M7 & $2(2 \%)$ & - & \\
\hline $\begin{array}{l}\text { NPM1 mutation } \\
\text { (positive) }\end{array}$ & $40(40 \%)$ & - & - \\
\hline $\begin{array}{l}\text { FLT3/ITD mutation } \\
\text { (positive) }\end{array}$ & $37(37 \%)$ & - & - \\
\hline \multicolumn{4}{|l|}{ lnRNA ANRIL } \\
\hline Mean \pm SD & $6.7 \pm 4.6$ & $1.4 \pm 1.2$ & $<0.001 *$ \\
\hline Median (Min. - Max.) & $5.7(0.5-20.7)$ & $0.9(0-6.5)$ & \\
\hline \multicolumn{4}{|l|}{ lnRNA SNHG14 } \\
\hline Mean \pm SD & $10.6 \pm 7.1$ & $1.4 \pm 1.2$ & $<0.001 *$ \\
\hline Median (Min. - Max.) & $8.4(0.1-29.2)$ & $0.9(0.4-7)$ & \\
\hline \multicolumn{4}{|l|}{ Clinical Remission } \\
\hline CR & $60(60 \%)$ & - & - \\
\hline NCR & $40(40 \%)$ & - & \\
\hline \multicolumn{4}{|l|}{ Relapse } \\
\hline No relapse & $64(64 \%)$ & - & - \\
\hline Relapse & $36(36 \%)$ & - & - \\
\hline \multicolumn{4}{|l|}{ Survival } \\
\hline Alive & $64(64 \%)$ & - & - \\
\hline Dead & $36(36 \%)$ & - & \\
\hline
\end{tabular}

$\chi^{2}$, Chi square test; U, Mann Whitney test; SD, Standard deviation; $\mathrm{p}, \mathrm{p}$ value for comparing between AML patients and Controls; *, Statistically significant at $\mathrm{p} \leq 0.05$

(Table 2).

On the other hand, InRNA SNHG14 was significantly associated with positive FLT3/ITD mutation $(p=0.023)$, while no association with NPM1 mutation $(p=0.221)$ in patients with AML (Table 2).

Target gene expression levels and clinical outcomes Response to treatment

As we studied the impact of the gene expression levels on the CR response, we found out that both high lnRNA ANRIL expression and high SNHG14 expression were significantly associated with higher relapse rate $(\mathrm{p}<0.001$ for ANRIL and $\mathrm{p}<0.001$ for SNHG14) (Table 2).

On univariate testing using age, WBC count, NPM1, FLT3/ITD, lnRNA ANRIL and InRNA SNHG14, it was found that FLT3/ITD (OR=3.667, 95\% CI: 10561-8.614, $\mathrm{p}=0.003)$, high lnRNA ANRIL expression $(\mathrm{OR}=4.896$ ,95\% CI: 2.044-11.728, $\mathrm{p}<0.001)$ and high lnRNA SNHG14 expression (OR=6 ,95\% CI: 2.453-14.678, $\mathrm{p}<0.001)$ were significantly linked to lower $C R$ rate compared with the low lnRNA expression (Table 4).

On multivariate logistic analysis, only FLT3/ITD (OR: 2.872, 95\% CI: 1.098-7.513, p=0.032), lnRNA ANRIL (OR: 3.449, 95\% CI: 1.324-8.985, $\mathrm{p}=0.011$ ), lnRNA SNHG14 (OR: 3.955, 95\% CI: 1.510-10.356, p=0.005) expression levels proved a statistically significant risk on complete remission response after induction therapy. On the contrary, other factors were not linked to treatment outcomes in AML patients (Table 5).

\section{Survival analysis}

High lnRNA ANRIL and high lnRNA SNHG14 expression levels were significantly linked to higher relapse rates compared with low expression levels (58\% vs $14 \%, p<0.001$ for $\operatorname{lnRNA}$ ANRIL and $58 \%$ vs $14 \%$, $\mathrm{p}<0.001$ for lnRNA SNHG14) (Table 2).

Survival analysis was predicted through KaplanMeier testing methodology to calculate the RFS and OS. According to target gene expressions, high lnRNAANRIL and high lnRNA SNHG14 expressions were associated with poor RFS $(p<0.001$ for ANRIL and $p<0.001$ for SNHG14) and shorter OS $(\mathrm{p}<0.001$ for ANRIL and $\mathrm{p}<0.001$ for SNHG14) (Table 2, Figure 3 and Figure 4).

A COX univariate and multivariate analyses were made for both RFS and OS. In the univariate analysis, the $\mathrm{CR}(\mathrm{HR}=3.570,95 \% \mathrm{CI}: 1.957-6.512, \mathrm{p}<0.001$ for RFS and $\mathrm{HR}=2.816$, 95\% CI: $1.439-5.510$, p:0.003 for OS), FLT3/ITD $(\mathrm{HR}=1.971,95 \% \mathrm{CI}: 1.109-3.503), \mathrm{p}=0.021$ for RFS and $\mathrm{HR}=2.440,95 \% \mathrm{CI}$ : $1.262-4.717$, p:0.008 for OS), InRNA ANRIL (HR=4.865, 95\%CI: 2.465-9.600, $\mathrm{p}<0.001$ for RFS and $\mathrm{HR}=4.579,95 \% \mathrm{CI}: 2.084-10.062$, $\mathrm{p}<0.001$ for OS), lnRNA SNHG14 (HR=5.682, 95\%CI: 2.812-11.483, $\mathrm{p}<0.001$ for RFS and $\mathrm{HR}=4.646,95 \% \mathrm{CI}$ : 2.114-10.209, p: $<0.001$ for OS) were significantly linked to shorter RFS and shorter OS (Table 4).

However, in the multivariate analysis, only lnRNA ANRIL (HR=3.504, 95\%CI: 1.662-7.387, $\mathrm{p}=0.001$ for RFS and $H R=3.353,95 \% \mathrm{CI}$ : $1.434-7.839, \mathrm{p}=0.005$ for OS) and lnRNA SNHG14 (HR=4.094, 95\%CI: 1.849 9.067, $\mathrm{p}=0.001$ for RFS and $\mathrm{HR}=3.094,95 \% \mathrm{CI}$ : 1.277 $7.494, p=0.012$ for OS) were the only significant risk factors independently impacting shorter RFS and shorter OS in AML patients (Table 5).

\section{Discussion}

lnRNAs were previously considered as "transcriptional noises", but as the research studies developed, it was revealed that $\ln$ RNAs have essential roles affecting genes expressions at so many levels; transcriptional, post-transcriptional and epigenetic levels. They were proved by numerous studies to participate in the 
Table 2. Clinical Criteria According to the Gene Expression Levels in AML Patients

\begin{tabular}{|c|c|c|c|c|}
\hline & \multicolumn{2}{|c|}{ InRNA ANRIL } & \multicolumn{2}{|c|}{ lnRNA SNHG14 } \\
\hline & Low $(\mathrm{n}=50)$ & High $(\mathrm{n}=50)$ & Low $(\mathrm{n}=50)$ & $\operatorname{High}(\mathrm{n}=50)$ \\
\hline \multicolumn{5}{|l|}{ Age } \\
\hline$<60$ & $38(76 \%)$ & $40(80 \%)$ & $40(80 \%)$ & $38(76 \%)$ \\
\hline$\geq 60$ & $12(24 \%)$ & $10(20 \%)$ & $10(20 \%)$ & $12(24 \%)$ \\
\hline$\chi^{2}(p)$ & \multicolumn{2}{|c|}{$0.233(0.627)$} & \multicolumn{2}{|c|}{$0.233(0.629)$} \\
\hline \multicolumn{5}{|l|}{ Gender } \\
\hline Male & $35(70 \%)$ & $32(64 \%)$ & $36(72 \%)$ & $31(62 \%)$ \\
\hline Female & $15(30 \%)$ & $18(36 \%)$ & $14(28 \%)$ & $19(38 \%)$ \\
\hline$\chi^{2}(\mathrm{p})$ & \multicolumn{2}{|c|}{$0.407(0.523)$} & \multicolumn{2}{|c|}{$1.131(0.288)$} \\
\hline \multicolumn{5}{|l|}{ WBC count $* 109 / 1$} \\
\hline Mean \pm SD & $41.9 \pm 53.2$ & $48.7 \pm 54.3$ & $43.5 \pm 47.4$ & $47.1 \pm 59.6$ \\
\hline Median (Min. - Max.) & $25.5(0.5-302)$ & $33(0.3-290)$ & $32(0.3-302)$ & $26(0.5-290)$ \\
\hline $\mathrm{U}(\mathrm{p})$ & \multicolumn{2}{|c|}{$1117.50(0.361)$} & \multicolumn{2}{|c|}{$1167.0(0.567)$} \\
\hline \multicolumn{5}{|l|}{ FAB classification } \\
\hline M0 & $9(18 \%)$ & $8(16 \%)$ & $8(16 \%)$ & $9(18 \%)$ \\
\hline M1 & $8(16 \%)$ & $8(16 \%)$ & $7(14 \%)$ & $9(18 \%)$ \\
\hline M2 & $7(14 \%)$ & $12(24 \%)$ & $7(14 \%)$ & $12(24 \%)$ \\
\hline M4 & $12(24 \%)$ & $8(16 \%)$ & $13(26 \%)$ & $7(14 \%)$ \\
\hline M5 & $13(26 \%)$ & $12(24 \%)$ & $14(28 \%)$ & $11(22 \%)$ \\
\hline M6 & $0(0 \%)$ & $1(2 \%)$ & $0(0 \%)$ & $1(2 \%)$ \\
\hline M7 & $1(2 \%)$ & $1(2 \%)$ & $1(2 \%)$ & $1(2 \%)$ \\
\hline$\chi^{2}(\mathrm{MCp})$ & \multicolumn{2}{|c|}{$3.425(0.822)$} & \multicolumn{2}{|c|}{$4.940(0.567)$} \\
\hline NPM1 mutation (positive) & $27(54 \%)$ & $13(26 \%)$ & $23(46 \%)$ & $17(34 \%)$ \\
\hline$\chi^{2}(\mathrm{p})$ & $8.167 *(0.004 *)$ & & $1.500(0.221)$ & \\
\hline FLT3/ITD mutation (positive) & $15(30 \%)$ & $22(44 \%)$ & $13(26 \%)$ & $24(48 \%)$ \\
\hline$\chi^{2}(\mathrm{p})$ & \multicolumn{2}{|c|}{$2.102(0.147)$} & \multicolumn{2}{|c|}{$5.191 *(0.023 *)$} \\
\hline \multicolumn{5}{|l|}{ Response to induction therapy } \\
\hline $\mathrm{CR}$ & $39(78 \%)$ & $21(42 \%)$ & $40(80 \%)$ & $20(40 \%)$ \\
\hline NCR & $11(22 \%)$ & $29(58 \%)$ & $10(20 \%)$ & $30(60 \%)$ \\
\hline$\chi^{2}(\mathrm{p})$ & \multicolumn{2}{|c|}{$13.500 *(<0.001 *)$} & \multicolumn{2}{|c|}{$16.667 *(<0.001 *)$} \\
\hline \multicolumn{5}{|l|}{ Relapse } \\
\hline No relapse & $43(86 \%)$ & $21(42 \%)$ & $43(86 \%)$ & $21(42 \%)$ \\
\hline Relapse & $7(14 \%)$ & $29(58 \%)$ & $7(14 \%)$ & $29(58 \%)$ \\
\hline$\chi^{2}(p)$ & \multicolumn{2}{|c|}{$21.007^{*}(<0.001 *)$} & \multicolumn{2}{|c|}{$21.007 *(<0.001 *)$} \\
\hline \multicolumn{5}{|l|}{ Survival } \\
\hline Survived & $42(84 \%)$ & $22(44 \%)$ & $42(84 \%)$ & $22(44 \%)$ \\
\hline Died & $8(16 \%)$ & $28(56 \%)$ & $8(16 \%)$ & $28(56 \%)$ \\
\hline$\chi^{2}(p)$ & \multicolumn{2}{|c|}{$17.361 *(<0.001 *)$} & \multicolumn{2}{|c|}{$17.361 *(<0.001 *)$} \\
\hline
\end{tabular}

$\chi^{2}$, Chi square test ; U, Mann Whitney test ; SD, Standard deviation; *, Statistically significant at $\mathrm{p} \leq 0.05$

development of cancers. Therefore, lnRNAs are regarded as future opportunities for diagnosis and treatment of malignant tumours (Wang and Wen, 2020).

Studies have shown that InRNAs play pivotal roles in pathogenesis of AML. For example, LINC01018 was found to be downregulated in AML and its upregulation inhibited AML cell growth and might provide therapeutic evidence in the future (Zhou et al., 2021) . On the other hand, lncRNA CD27 Antisense RNA 1 (CD27-AS1) was found to be overexpressed in AML and might be a potential prognostic marker for AML in the future
(Tao et al., 2021) . In the present study, we revealed that InRNAANRIL and SNHG14 were overexpressed in AML patients, suggesting they play a role in AML development and prognosis. Similarly, previous research reported that InRNA ANRIL and InRNA SNHG14 were overexpressed in AML patients' samples compared to healthy controls (Tan et al., 2021; Wang et al., 2021).

Regarding lnRNA ANRIL, in this study, lnRNA ANRIL was capable of differentiating AML patients from controls. Moreover, we demonstrated that it was associated with high FLT3-ITD and low NPM-1 mutations in AML 

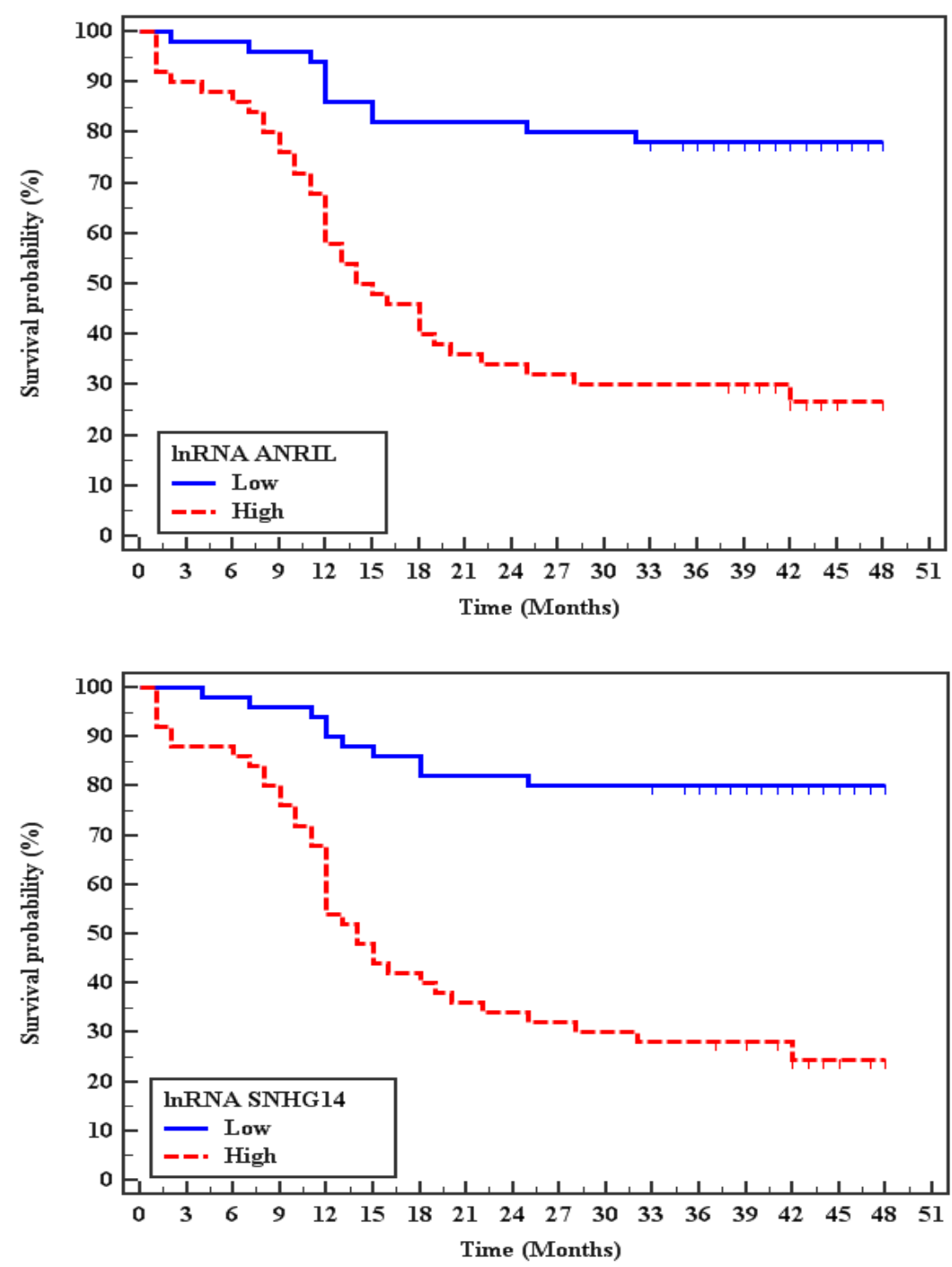

Figure 3. Kaplan-Meier Survival Curves for the Comparison of RFS between AML Patients According to the Level of Expression of A) lnRNA ANRIL and B) lnRNA SNHG14

Table 4. Univariate Analysis of Prognostic Value of AML Patients

\begin{tabular}{lcccccc}
\hline & \multicolumn{2}{c}{ CR } & \multicolumn{2}{c}{ RFS } & \multicolumn{2}{c}{ OS } \\
& $\mathrm{p}$ & OR $(95 \%$ C.I $)$ & $\mathrm{p}$ & HR $(95 \% \mathrm{C} . \mathrm{I})$ & $\mathrm{p}$ & HR $(95 \%$ C.I $)$ \\
\hline Age $>60$ & 0.921 & $1.050(0.401-2.750)$ & 0.9 & $1.044(0.531-2.051)$ & 0.661 & $1.184(0.557-2.517)$ \\
WBC count & 0.963 & $1.000(0.993-1.008)$ & 0.586 & $1.001(0.996-1.006)$ & 0.515 & $1.002(0.996-1.007)$ \\
CR vs NCR & & & $<0.001^{*}$ & $3.570(1.957-6.512)$ & $0.003^{*}$ & $2.816(1.439-5.510)$ \\
NPM1 & 0.677 & $0.840(0.370-1.908)$ & 0.437 & $0.790(0.436-1.433)$ & 0.2 & $0.629(0.310-1.279)$ \\
FLT3/ITD & $0.003^{*}$ & $3.667(1.561-8.614)$ & $0.021^{*}$ & $1.971(1.109-3.503)$ & $0.008^{*}$ & $2.440(1.262-4.717)$ \\
lnRNA ANRIL & $<0.001^{*}$ & $4.896(2.044-11.728)$ & $<0.001^{*}$ & $4.865(2.465-9.600)$ & $<0.001^{*}$ & $4.579(2.084-10.062)$ \\
lnRNA SNHG14 & $<0.001^{*}$ & $6.000(2.453-14.678)$ & $<0.001^{*}$ & $5.682(2.812-11.483)$ & $<0.001 *$ & $4.646(2.114-10.209)$ \\
\hline
\end{tabular}

HR, Hazard ratio; OR, Odd`s ratio; C.I, Confidence interval; LL, Lower limit; UL, Upper Limit; *, Statistically significant at $\mathrm{p} \leq 0.05$

patients when compared to controls and significantly lower CR, RFS and OS in AML patients with high expression of
ANRIL compared to AML patients with low expression of ANRIL. Previous studies showed that downregulation

Table 3. Agreement (Sensitivity, Specificity) for Different Parameters to Predict AML Patients vs Control

\begin{tabular}{lcccccccc}
\hline & AUC & P & $95 \%$ C.I & Cut off & Sensitivity & Specificity & PPV & NPV \\
\hline lnRNA ANRIL & $0.924^{*}$ & $<0.001^{*}$ & $0.887-0.960$ & $>2.51$ & 83 & 90 & 89.2 & 84.1 \\
lnRNA SNHG14 & $0.919^{*}$ & $<0.001^{*}$ & $0.872-0.965$ & $>3.86$ & 87 & 97 & 96.7 & 88.2 \\
\hline
\end{tabular}

AUC, Area Under a Curve; p value, Probability value; CI, Confidence Intervals; NPV, Negative predictive value; PPV, Positive predictive value *, Statistically significant at $\mathrm{p} \leq 0.05$. 

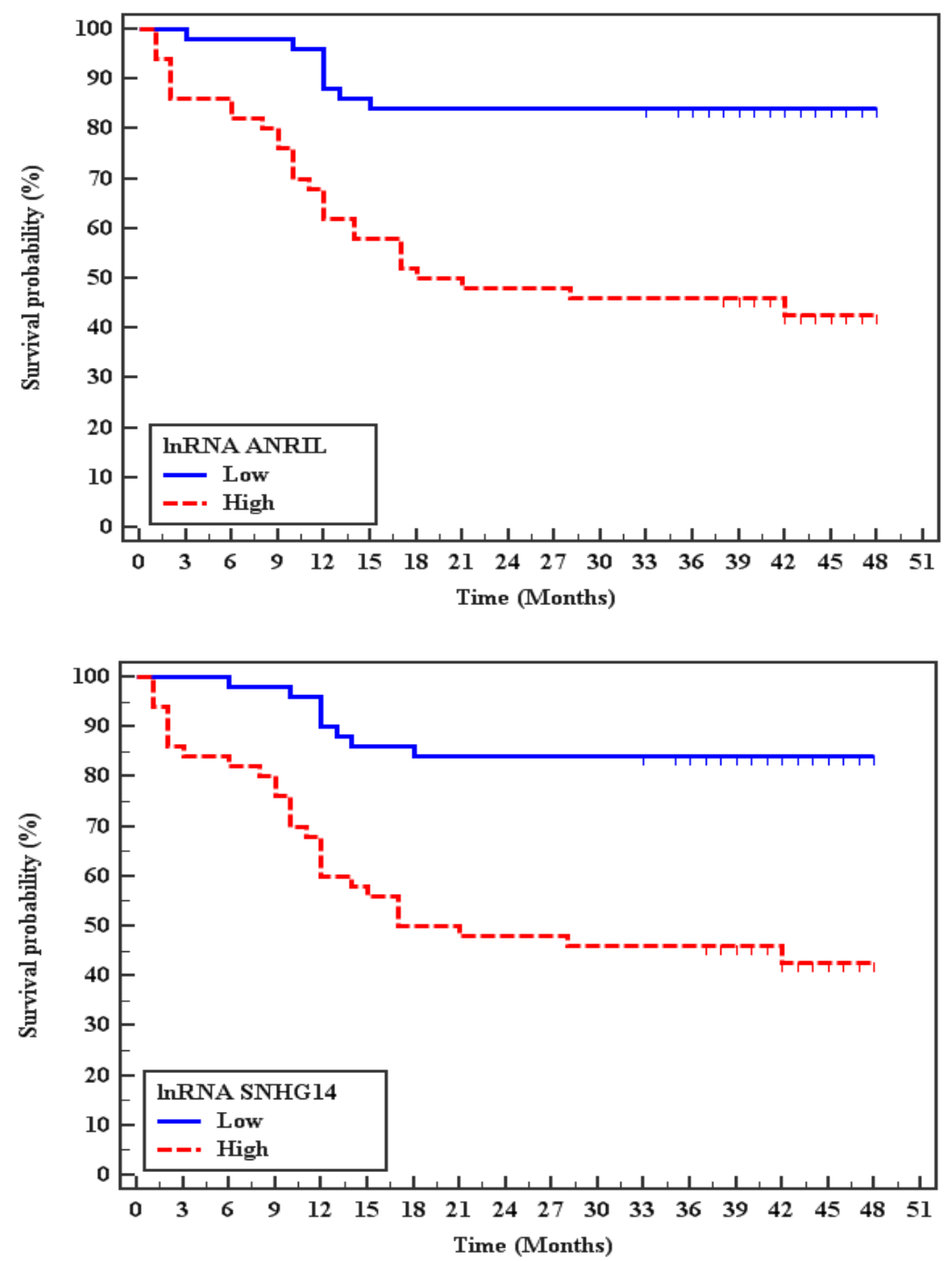

Figure 4. Kaplan-Meier Survival Curves for the Comparison of OS was Analysed in CN-AML According to the Different Expression Levels of A) lnRNA ANRIL and B)InRNA SNHG14

of ANRIL suppressed AML cell proliferation, invasion, and increased apoptosis (Wang et al., 2020). Similarly, a study carried out by Tan et al., (2021) proved that lnRNA ANRIL was significantly associated with AML development, FLT3 mutation and low CR, RFS and OS. Therefore, we can say that lnRNA ANRIL is associated with poor prognosis in AML. The explanation for this could be due to the fact that ANRIL inhibits the tumor suppressor gene p15 (INK4B) leading to malignant cells proliferation (Zhang et al., 2018b). Also, it could be due to InRNA ANRIL suppresses AdipoR1 that accelerates AML malignant cells survival and proliferation (Sun et al., 2018). Finally, ANRIL might cause chemoresistance in AML patients by enhancing ATP-binding subfamily C member 1 leading to drug resistance and poor RFS and OS (Zhang et al., 2018b).

Previous research works studied SNHG14 in other malignancies (Xu et al., 2017; Liu et al., 2018a; Sun et al., 2018; Zhang et al., 2018a; Ma et al., 2019). For example, $\mathrm{Xu}$ et al., (2017) reported that ANRIL enhance malignant cell proliferation and inhibited apoptosis in breast cancer via sponging miR-199a. Similarly, Liu (2018) studied ANRIL in gastric cancer and reported that ANRIL inhibition inhibited malignant cell viability and ability

Table 5. Multivariate Analysis of Prognostic Value of AML Patients

\begin{tabular}{|c|c|c|c|c|c|c|}
\hline & \multicolumn{2}{|r|}{$\mathrm{CR}$} & \multicolumn{2}{|r|}{ RFS } & \multicolumn{2}{|r|}{ OS } \\
\hline & $\mathrm{p}$ & OR $(95 \%$ C.I $)$ & $\mathrm{p}$ & HR (95\%C.I) & $\mathrm{p}$ & HR (95\%C.I) \\
\hline CR vs NCR & & & 0.528 & $1.264(0.611-2.617)$ & 0.993 & $1.003(0.454-2.218)$ \\
\hline FLT3/ITD & $0.032 *$ & $2.872(1.098-7.513)$ & 0.856 & $1.059(0.571-1.964)$ & 0.192 & $1.593(0.791-3.207)$ \\
\hline lnRNA ANRIL & $0.011 *$ & $3.449(1.324-8.985)$ & $0.001 *$ & $3.504(1.662-7.387)$ & $0.005^{*}$ & $3.353(1.434-7.839)$ \\
\hline lnRNA SNHG14 & $0.005 *$ & $3.955(1.510-10.356)$ & $0.001 *$ & $4.094(1.849-9.067)$ & $0.012 *$ & $3.094(1.277-7.494)$ \\
\hline
\end{tabular}

HR, Hazard ratio; OR,: Odd`s ratio; C.I, Confidence interval; LL, Lower limit; UL, Upper Limit; \#, All variables with $p<0.05$ was included in the multivariate; *, Statistically significant at $\mathrm{p} \leq 0.05$ 
to invade normal tissues and stimulated apoptosis via miR-99a- mediated inhibition of B-lymphoma Moloney murine leukemiavirus insertion region (Döhner et al., 2015; Liu et al., 2018a). These findings hypothesise strong relationship between ANRIL and tumorigenesis.

Regarding lnRNA SNHG14, in this study, lnRNA SNHG14 was capable of differentiating AML patients from controls. Moreover, we demonstrated that it was associated with high FLT3-ITD and low NPM-1 mutations in AML patients when compared to controls and significantly lower CR, RFS and OS in AML patients with high expression of SNHG14 compared to AML patients with low expression of SNHG14. Previous studies showed that SNHG14 was overexpressed in bone marrow samples of AML patients compared to controls suggesting that it might function as an oncogene in AML development (Wang et al., 2021). According to Wang et al., (2021), the possible explanation for this association between lnRNA SNHG14 and AML occurrence and poor prognosis might be that SNHG14 was a sponge for miR-193b-3p and that miR-193b-3p was negatively regulated by SNHG14. miR-193b-3p was reported to be under expressed in AML patients demonstrating that it might have an anti-tumour effect on AML malignant cells (Xu et al., 2017; Bhayadia et al., 2018; Liu et al., 2018a; Zhang et al., 2018a; Zhang et al., 2018b; Wang et al., 2021).

Previous research studied ANRIL in other malignancies (Liu et al., 2018b; Di et al., 2019; Ji et al., 2019; Pu et al., 2019). For example, it was reported that SNHG14 increased cervical cancer progression by sponging miR-206 (Ji et al., 2019). In addition, Liu (2018) showed that SNHG14 targeted miR-145 modulating SOx9 expression, thus inducing gastric cancer (Liu et al., 2018b). These results suggested that SNHG14 might have a role as an oncogene in AML development via clearing miR193-3p.

Our study had some limitations that worth mentioning to overcome in the coming research works. Firstly, the number of AML patients was limited, and their follow up period was relatively short. Secondly, we assessed the lnRNA ANRIL and SNHG14 gene expressions at the baseline level only without reassessing them after treatment to check the difference if there was a difference in the treatment outcomes.

In conclusion, InRNA SNHG14 and InRNA ANRIL showed high expression levels in AML bone marrow samples and were remarkably associated with lower CR, RFS and OS, favouring poor prognostic significance in AML. This suggests that both InRNA ANRIL and lnRNA SNHG14 could be used in the future as prognostic biomarkers that aid in treatment decisions and follow up of AML patients.

\section{Author Contribution Statement}

MG, OG and RA contributed to the design and implementation of the research, to the analysis of the results and to the writing of the manuscript.

\section{Acknowledgments}

Authors would like to thank all the patients and their families for participating in this project.

Consent for publication The Authors grant the Publisher permission to publish this work.

Availability of data and material The data that support the findings of this study are available from the corresponding author upon request.

\section{Conflicts of interest}

Authors declare no conflict of interest.

\section{References}

Abo Elwafa R, Gamaleldin M, Ghallab O (2019). The clinical and prognostic significance of FIS1, SPI1, PDCD7 and Ang2 expression levels in acute myeloid leukemia. Cancer Genet, 2019, 233-234, 84-95.

Bhan A, Soleimani M, Mandal SS (2017). Long noncoding RNA and cancer: A New Paradigm. Cancer Res, 77, 3965-81.

Bhayadia R, Krowiorz K, Haetscher N, et al (2018). Endogenous tumor suppressor microRNA-193b: Therapeutic and Prognostic Value in Acute Myeloid Leukemia. J Clin Oncol, 36, 1007-16.

Congrains A, Kamide K, Ohishi M, et al (2013). ANRIL: molecular mechanisms and implications in human health. Int J Mol Sci, 14, 1278-92.

Cruz-Miranda GM, Hidalgo-Miranda A, Bárcenas-López DA, et al (2019). Long non-coding RNA and acute leukemia. Int $J$ Mol Sci, 20.

Di W, Weinan X, Xin L, et al (2019). Long noncoding RNA SNHG14 facilitates colorectal cancer metastasis through targeting EZH2-regulated EPHA7. Cell Death Dis, 10, 514.

Döhner H, Estey EH, Amadori S, et al (2010). Diagnosis and management of acute myeloid leukemia in adults: recommendations from an international expert panel, on behalf of the European LeukemiaNet. Blood, 115, 453-74.

Döhner H, Weisdorf DJ, Bloomfield CD (2015). Acute Myeloid Leukemia. N Engl J Med, 373, 1136-52.

Ferrè F, Colantoni A, Helmer-Citterich M (2016). Revealing protein-IncRNA interaction. Brief Bioinform, 17, 106-16.

Gamaleldin MA, Imbaby SAE (2021). The role of tumor necrosis factor receptor superfamily member 4 (TNFRSF4) gene expression in diagnosis and prognosis of acute myeloid leukemia. Mol Biol Rep, 48, 6831-43.

Gugnoni M, Ciarrocchi A (2019). Long noncoding RNA and epithelial mesenchymal transition in cancer. Int JMol Sci, 20.

Hao YR, Zhang DJ, Fu ZM, et al (2019). Long non-coding RNA ANRIL promotes proliferation, clonogenicity, invasion and migration of laryngeal squamous cell carcinoma by regulating miR-181a/Snai2 axis. Regen Ther, 11, 282-9.

Jathar S, Kumar V, Srivastava J, et al (2017). Technological developments in IncRNA biology. Adv Exp Med Biol, 1008, 283-323.

Ji N, Wang Y, Bao G, et al (2019). LncRNA SNHG14 promotes the progression of cervical cancer by regulating miR-206/ YWHAZ. Pathol Res Pract, 215, 668-75.

Kadia TM, Ravandi F, Cortes J, et al (2016). New drugs in acute myeloid leukemia. Ann Oncol, 27, 770-8.

Kuendgen A, Germing U (2009). Emerging treatment strategies for acute myeloid leukemia (AML) in the elderly. Cancer Treat Rev, 35, 97-120.

Liu P, Zhang M, Niu Q, et al (2018a). Knockdown of long noncoding RNAANRIL inhibits tumorigenesis in human gastric cancer cells via microRNA-99a-mediated down-regulation 
of BMI1. Braz J Med Biol Res, 51, e6839.

Liu Z, Yan Y, Cao S, et al (2018b). Long non-coding RNA SNHG14 contributes to gastric cancer development through targeting miR-145/SOX9 axis. J Cell Biochem, 119, 6905-13.

Livak KJ, Schmittgen TD (2001). Analysis of relative gene expression data using real-time quantitative PCR and the 2(-Delta Delta C(T)) Method. Methods, 25, 402-8.

Ma Y, Zhang H, Li G, et al (2019). LncRNA ANRIL promotes cell growth, migration and invasion of hepatocellular carcinoma cells via sponging miR-144. Anticancer Drugs, 30, 1013-21.

Pasmant E, Laurendeau I, Héron D, et al (2007). Characterization of a germ-line deletion, including the entire INK4/ARF locus, in a melanoma-neural system tumor family: identification of ANRIL, an antisense noncoding RNA whose expression coclusters with ARF. Cancer Res, 67, 3963-9.

Prada-Arismendy J, Arroyave JC, Röthlisberger S (2017). Molecular biomarkers in acute myeloid leukemia. Blood Rev, 31, 63-76.

Pu J, Wei H, Tan C, et al (2019). Long noncoding RNA SNHG14 facilitates hepatocellular carcinoma progression through regulating miR-4673/SOCS1. Am J Transl Res, 11, 5897904.

Shallis RM, Wang R, Davidoff A, et al (2019). Epidemiology of acute myeloid leukemia: Recent progress and enduring challenges. Blood Rev, 36, 70-87.

Sun LY, Li XJ, Sun YM, et al (2018). LncRNAANRIL regulates AML development through modulating the glucose metabolism pathway of AdipoR1/AMPK/SIRT1. Mol Cancer, 17, 127.

Tan Z, Zhu K, Yin Y, et al (2021). Long non-coding RNA ANRIL is a potential indicator of disease progression and poor prognosis in acute myeloid leukemia. Mol Med Rep, 23.

Tao Y, Zhang J, Chen L, et al (2021). LncRNA CD27-AS1 promotes acute myeloid leukemia progression through the miR-224-5p/PBX3 signaling circuit. Cell Death Dis, 12, 510.

Wang CH, Li QY, Nie L, et al (2020). LncRNAANRIL promotes cell proliferation, migration and invasion during acute myeloid leukemia pathogenesis via negatively regulating miR-34a. Int J Biochem Cell Biol, 119, 105666.

Wang GF, Wen LN (2020). LncRNA SNHG14 promotes proliferation of endometrial cancer through regulating microRNA-655-3p. Eur Rev Med Pharmacol Sci, 24, 10410-8.

Wang X, Li W, Chen Y, et al (2021). Long non-coding RNA SNHG14 affects the proliferation and apoptosis of childhood acute myeloid leukaemia cells by modulating the miR-193b-3p/MCL1 axis. Mol Med Rep, 23.

Wu K, Li J, Qi Y, et al (2019). SNHG14 confers gefitinib resistance in non-small cell lung cancer by up-regulating ABCB1 via sponging miR-206-3p. Biomed Pharmacother, 116, 108995.

Xu ST, Xu JH, Zheng ZR, et al (2017). Long non-coding RNA ANRIL promotes carcinogenesis via sponging miR-199a in triple-negative breast cancer. Biomed Pharmacother, 96, 14-21.

Zhang LM, Ju HY, Wu YT, et al (2018a). Long non-coding RNA ANRIL promotes tumorgenesis through regulation of FGFR1 expression by sponging miR-125a-3p in head and neck squamous cell carcinoma. Am J Cancer Res, 8, 2296-310.

Zhang YY, Li M, Xu YD, et al (2019a). LncRNA SNHG14 promotes the development of cervical cancer and predicts poor prognosis. Eur Rev Med Pharmacol Sci, 23, 3664-71.

Zhang Z, Feng L, Liu P, et al (2018b). ANRIL promotes chemoresistance via disturbing expression of $\mathrm{ABCC} 1$ by regulating the expression of Let-7a in colorectal cancer. Biosci Rep, 38.
Zhang Z, Wang Y, Zhang W, et al (2019b). Long non-coding RNA SNHG14 exerts oncogenic functions in non-small cell lung cancer through acting as an miR-340 sponge. Biosci Rep, 39.

Zhou H, Shi P, Jia X, et al (2021). Long non-coding RNA LINC01018 inhibits the progression of acute myeloid leukemia by targeting miR-499a-5p to regulate PDCD4. Oncol Lett, 22, 541.

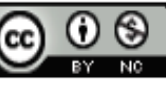

This work is licensed under a Creative Commons AttributionNon Commercial 4.0 International License. 